\title{
Impact of COVID-19 on chronic migraine treated with erenumab: a case report
}

\author{
Alberto Grassini ${ }^{1}$ (D) Andrea Marcinnò ${ }^{1} \cdot$ Fausto Roveta $^{1} \cdot$ Erica Gallo ${ }^{1} \cdot$ Aurora Cermelli $^{1} \cdot$ Silvia Boschi $^{1}$. \\ Elisa Rubino ${ }^{1} \cdot$ Innocenzo Rainero ${ }^{1}$
}

Received: 14 May 2021 / Accepted: 17 May 2021 / Published online: 21 May 2021

(C) Fondazione Società Italiana di Neurologia 2021

\begin{abstract}
Background Headache is a frequent symptom of the novel coronavirus 19 disease (COVID-19). To date, there are limited information on how COVID-19 affects migraine and its treatment.

Case description A 47-year-old patient, suffering from chronic migraine and medication-overuse headache, in September 2020 started erenumab at $70 \mathrm{mg}$ once monthly. Two months later, monthly migraine days decreased from 20 to 5 . On the third month, the patient developed mild COVID-19 symptoms, experiencing extreme fatigue, hyposmia, and attention deficit, resulting positive for SARSCov-2 RNA. A significant increase in migraine attacks frequency was reported. Brain MRI and EEG were normal. Erenumab was increased to $140 \mathrm{mg} / \mathrm{month}$, and attacks decreased to 3 monthly migraine days and remained stable. All the headaches experienced by our patient during the infection fulfilled the criteria of the migraine attacks, without tensive-like features.

Conclusion We report the first case showing the effects of SARS-CoV-2 infection in a patient with chronic migraine and medication-overuse headache treated with erenumab. Our case description suggests that inflammatory processes induced by SARS-CoV-2 infection may increase the frequency of migraine attacks, probably through an activation of the trigeminovascular system. Whether treatment with CGRP receptor antagonist may influence COVID is still debated. Additional studies regarding anti-CGRP monoclonal antibodies in COVID-19 patients are warranted.
\end{abstract}

Keywords Chronic migraine $\cdot$ Erenumab $\cdot$ COVID-19 $\cdot$ SARS-Cov-2 $\cdot$ CGRP

\section{Introduction}

Since the beginning of the pandemic, headache was reported as one of the most prevalent symptoms in patients with novel coronavirus disease 19 (COVID-19). An observational study of 1420 patients with mild or moderate disease showed that headache was the most common symptom, affecting more than $70.3 \%$ of subjects [1]. Clinical characteristics of headache in COVID-19 vary greatly. Generally, headache is bilateral, mainly frontal, and with pressing quality. The majority of patients fulfills the International Classification of Headache Disorders, 3rd edition (ICDH-3) criteria for "Headache attributed to systemic viral infection" [2]; however approximately

Alberto Grassini

alberto.grassini@unito.it

1 Department of Neuroscience "Rita Levi Montalcini", Headache Center, Neurology I, University of Torino, Via Cherasco 15, 10126 Torino, Italy
$25 \%$ and $50 \%$ of patients also fulfill criteria for migraine and tension-type headache, respectively [3].

At present, the effect of COVID-19 in patients with migraine has been scarcely investigated. A cross-sectional study on severe acute respiratory coronavirus 2 (SARS-CoV-2)positive patients showed that individuals with a previous diagnosis of migraine tend to present with earlier, longer, and more intense headaches [4]. Additional data regarding the impact of COVID-19 on migraine are needed.

The purpose of this case report is to describe the impact of SARS-CoV-2 infection on the clinical characteristics of headache in a 47-year-old male patient with chronic migraine and medication-overuse headache who developed COVID-19 while on prophylactic treatment with the anti-CGRP monoclonal antibody erenumab.

\section{Case report}

The patient first presented in our outpatient department at age 41 . Recurrent headache attacks were reported since the age of 8 , with 
progressive worsening in frequency and intensity in the last years. The attacks were characterized by unilateral pulsating pain of moderate-severe intensity, localized in the orbital region with fronto-temporal irradiation, often associated with photo/ phonophobia, accompanied by nausea without vomiting, lasting up to 24-72 h, frequently triggered by psychological stress and physical activity. The patient reported no aura or trigeminoautonomic symptoms. No familiarity for migraine was reported, and medical history was unremarkable. In the last 2 years, reported monthly migraine days (MMD) were 20 , and monthly acute migraine-specific medication days (MSMD) were 15 . The patient fulfilled the ICHD-3 criteria for chronic migraine and medication-overuse headache [3].

Because of the constant increase of assumption of symptomatic drugs, in 2019, the patient was hospitalized for a week in order to perform a detoxification therapy, but the frequency of migraine attacks remained high (MMD 16). Previous prophylactic drugs (topiramate, timolol, atenolol, amitriptyline, and fluoxetine) were reported to be useless; therefore, in September 2020, a therapy with the anti-CGRP receptor monoclonal antibody erenumab, at dosage of $70 \mathrm{mg}$ per month, was prescribed. At baseline, Migraine Disability Assessment Test (MIDAS) and Headache Impact Test (HIT6) scores were 69 and 67, respectively. In the following 2 months, the patient referred a significant decrease of both frequency and intensity of migraine attacks, with only $5 \mathrm{mi}-$ graine days in the second month and a significant reduction in acute antimigraine drug assumption.

In the first days of November 2020, the patient developed hyposmia and intense fatigue. A nasopharyngeal swab test was performed, resulting positive for SARS-CoV-2 RNA. Fever or upper airway inflammatory symptoms were never reported. The swab got negative on November 24. During this period, the patient referred a significant worsening of migraine (MMD 15 in November) as well as episodes' intensity. In addition, the patient experienced mild neurocognitive symptoms, like attention and memory impairment. MIDAS and HIT-6 scores were 8 and 64, respectively. Headache attacks were reported to be more severe in intensity, but remaining clinical features were unchanged. The patient reported the assumption of several acute antimigraine drugs without significant relief of headache attacks. Mini Mental Examination score was $28 / 30$, revealing only a mild attention deficit. Physical and neurological examinations, blood tests, electroencephalogram, and MRI scan of the brain resulted normal.

Erenumab was increased to $140 \mathrm{mg} / \mathrm{month}$ at the beginning of December, and monthly migraine attacks decreased to 7 and then to 3 in the following months (Fig. 1). At the end of January, the patient referred a subjective reduction of fatigue and attention impairment, and all neuropsychological tests were normal. No significant side effects related to erenumab therapy were reported, both before and after SARS-CoV-2 infection.

\section{Discussion}

To the best of our knowledge, we report the first case showing the effects of SARS-CoV-2 infection in a patient with chronic migraine and medication-overuse headache treated with erenumab, an anti-CGRP receptor monoclonal antibody. A previous case report described the effects of COVID-19 on a 58 -year-old woman suffering from both chronic migraine and multiple sclerosis treated with fremanezumab and fingolimod [5]. The patient showed a significant worsening of headache attacks during infection. However, she also developed pneumonia and reported several clinical symptoms suggestive of meningoencephalitis due to SARS-CoV-2.

The exact mechanisms explaining increase of migraine attacks during SARS-CoV-2 infection in our patient are still to be elucidated. Potential explanations include
Fig. 1 The time course of Monthly Migraine Days since the beginning of erenumab therapy, during the SARS-CoV-2 infection and after increase in drug dosage

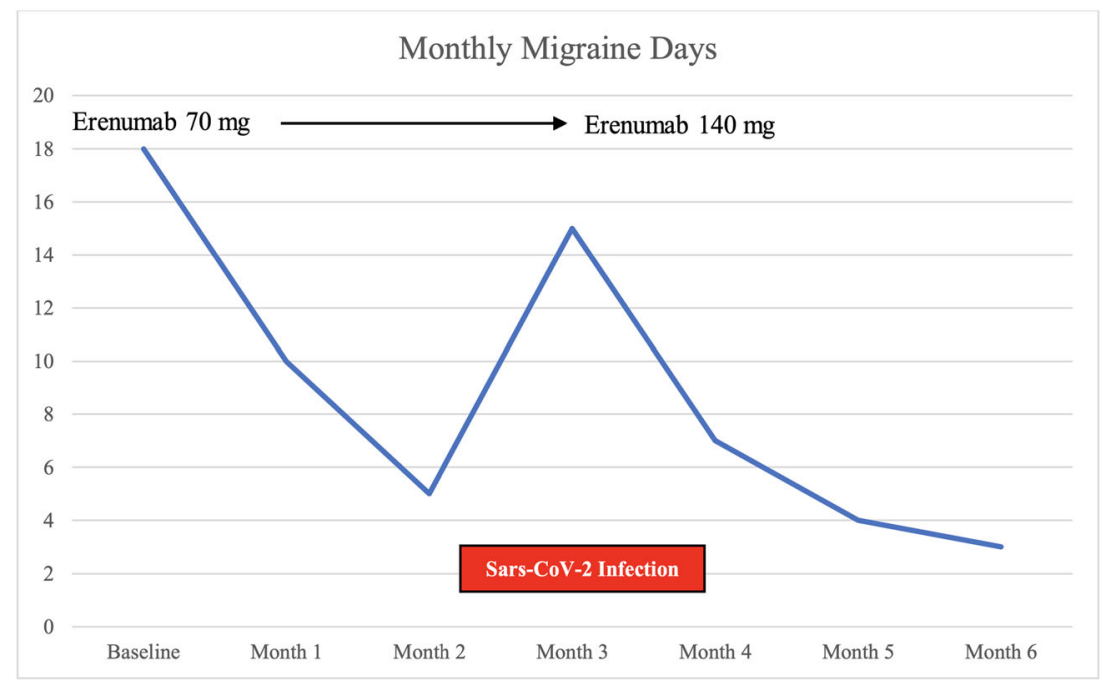


meningoencephalitis due to direct brain invasion by the viral agent, metabolic encephalopathy, and the cytokine release syndrome (CRS) [6]. The first two hypotheses seem unlikely since our patient developed a mild COVID-19 and neurologic investigations were normal. On the contrary, increased cytokine concentrations (the "cytokine storm") are the most plausible explanation of migraine worsening. In patients with severe SARS-CoV-2 infection, increased plasma concentrations of several pro-inflammatory molecules, as interferon gamma, interleukin-2, interleukin-6, tumor necrosis factor alpha, interleukin-8, and interleukin-10, were described [7]. Several studies in experimental animals as well as genetic and biochemical studies in humans suggested a role for pro-inflammatory cytokines in migraine pathophysiology $[8,9]$.

Our patient reported also mild neurocognitive symptoms lasting approximately 2 months and resolving without specific treatment. Several post-infectious complications of COVID19 affecting cognitive function (the so-called brain fog) have been described [10]. Mechanisms that may contribute to COVID19-associated neurocognitive symptoms are, at present, unclear. Additional studies are needed in order to better elucidate prevalence of neurocognitive symptoms, spectrum of clinical involvement, natural history, and therapeutic strategies in these patients.

In conclusion, our case report showed that SARS-CoV-2 infection in a patient treating chronic migraine and medication-overuse headache with erenumab induced a significant increase in the frequency of migraine attacks. Increase in drug dosage significantly reduced this phenomenon. Additional studies in migraine patients treated with antiCGRP antibodies during COVID-19 are needed.

Data availability AG has full access to all the data in the study and takes responsibility for the integrity of the data.

\section{Declarations}

Conflict of interests The authors declare no competing interests.

Ethical approval All procedures were performed in accordance with the local ethics committee and with the ethical standards laid down in the 1964 Declaration of Helsinki and its later amendments.

Informed consent Written informed consent was obtained from the subject participating in the study.

\section{References}

1. Lechien JR, Chiesa-Estomba CM, Place S, van Laethem Y, Cabaraux P, Mat Q, Huet K, Plzak J, Horoi M, Hans S, Rosaria
Barillari M, Cammaroto G, Fakhry N, Martiny D, Ayad T, Jouffe L, Hopkins C, Saussez S, COVID-19 Task Force of YO-IFOS, Blecic S, de Siati DR, Leich P, Souchay C, Rossi C, Journe F, Hsieh J, Ris L, el Afia F, Harmegnies B, Distinguin L, Chekkoury-Idrissi Y, Circiu M, Lavigne P, Lopez Delgado I, Calvo-Henriquez C, Falanga C, Coppee F, le Bon SD, Rodriguez A, Dequanter D, Cornelis JP, Vergez S, Koenen L, Giuditta M, Molteni G, Tucciarone M, Radulesco T, Khalife M, Fourneau AF, Cherifi S, Manto M, Michel J, Radulesco T, Molteni G, Tucciarone M, Mannelli G, Cantarella G (2020) Clinical and epidemiological characteristics of 1420 European patients with mildto-moderate coronavirus disease 2019. J Intern Med 288:335-344. https://doi.org/10.1111/joim.13089

2. Headache Classification Committee of the International Headache Society (IHS) (2018) The International Classification of Headache Disorders, 3rd edition. Cephalalgia 38:1-211. https://doi.org/10. 1177/0333102417738202

3. Lopez JT, Garcia-Azorin D, Planchuelo-Gomez A et al (2020) Phenotypic characterization of acute headache attributed to SARS-CoV-2: an ICHD-3 validation study on 106 hospitalized patients. Cephalalgia 40:1432-1442. https://doi.org/10.1177/ 0333102420965146

4. Membrilla JA, De Lorenzo I, Sastre M et al (2020) Headache as a cardinal symptom of coronavirus disease 2019: a cross-sectional study. Headache 60:2176-2191. https://doi.org/10.1111/head. 13967

5. Arca KN, Starling AJ (2020) Treatment-refractory headache in the setting of COVID-19 pneumonia: migraine or meningoencephalitis? Case Report. SN Compr Clin Med:1-4. https://doi.org/10. 1007/s42399-020-00369-y

6. Tolebeyan AS, Zhang N, Cooper V, Kuruvilla DE (2020) Headache in patients with severe acute respiratory syndrome coronavirus 2 infection: a narrative review. Headache 60:2131-2138. https://doi.org/10.1111/head.13980

7. Liu J, Li S, Liu J, Liang B, Wang X, Wang H, Li W, Tong Q, Yi J, Zhao L, Xiong L, Guo C, Tian J, Luo J, Yao J, Pang R, Shen H, Peng C, Liu T, Zhang Q, Wu J, Xu L, Lu S, Wang B, Weng Z, Han C, Zhu H, Zhou R, Zhou H, Chen X, Ye P, Zhu B, Wang L, Zhou W, He S, He Y, Jie S, Wei P, Zhang J, Lu Y, Wang W, Zhang L, Li L, Zhou F, Wang J, Dittmer U, Lu M, Hu Y, Yang D, Zheng X (2020) Longitudinal characteristics of lymphocyte responses and cytokine profiles in the peripheral blood of SARS-CoV-2 infected patients. EBioMedicine 55:102763. https://doi.org/10.1016/j. ebiom.2020.102763

8. Rainero I, Grimaldi LM, Salani G et al (2004) Association between the tumor necrosis factor-alpha $-308 \mathrm{G} / \mathrm{A}$ gene polymorphism and migraine. Neurology 62:141-143. https://doi.org/10.1212/01. WNL.0000101717.16799.8F

9. Edvinsson L, Agmund Haanes K, Warfvinge K (2019) Does inflammation have a role in migraine. Nat Rev Neurol 15:483-490. https://doi.org/10.1038/s41582-019-0216-y

10. Woo MS, Malsy J, Pottgen J et al (2020) Frequent neurocognitive deficits after recovery from mild COVID-19. Brain Commun 2: fcaa205. https://doi.org/10.1016/j.msard.2021.102984

Publisher's note Springer Nature remains neutral with regard to jurisdictional claims in published maps and institutional affiliations. 